\title{
Use of rating scales by consultant psychiatrists
}

\author{
KARL RICE, Senior Registrar, Cefn Coed Hospital, Swansea SA2 0GH; and \\ Peter Donnelly, Senior Registrar, St David's Hospital, Carmarthen
}

The number and diversity of rating scales and tests of psychiatric status has increased dramatically in the last 20 to 30 years. Tests are in screening, e.g. for dementia the Mini Mental State Examination and the Abbreviated Mental Test; to aid clarification of the diagnosis in difficult cases, e.g. Walton-Black New Word Learning Test, to differentiate between depression and dementia; and in assessing severity of symptoms, e.g. Hamilton Depression Rating Scale.

The number of rating scales for the assessment of the severity of depression has increased from the original Hamilton to at least nine specific instruments currently available. In addition, there are at least six general instruments which include a depression scale, e.g. the depression scale of the Minnesota Multiphasic Personality Inventory.

When one looks at clinical practice and research work performed with depressed cohorts, the striking feature is the wide diversity in the use of rating scales. The lack of uniformity does not facilitate comparison between studies. This point was addressed at the Dahlem Conference in 1982 (Kupfer \& Rush, 1983). This conference was composed of a group of clinical investigators of depression. When considering the severity of illness the group recommended the use of at least one clinician rated method with proven reliability and validity. They considered that a self-report symptom scale may also be helpful but not essential. There was no consensus about which symptom severity rating scales should be used.

There is considerable criticism of the usefulness of rating scales, for example, that the information obtained is limited, yet unwarranted assumptions are made due to the apparent objectivity of the scales. Some psychologists regard extensive use of tests as a measure of lack of sophistication. However, established research methodology would be impossible without the use of such instruments. Use of psychological tests and rating scales by consultant psychiatrists has not been explored to date.

The aim of this study was to determine the most frequently used rating scales by a sample of consultant psychiatrists and to determine the characteristics of those using them.

Our hypothesis was that consultants in teaching centres are more likely to use rating scales.

\section{The study}

We used a two-stage postal survey. All consultants employed in the South West Thames region in summer 1989 were sent a questionnaire. They were asked to rate on a 1 to 6 scale their familiarity and use of 32 common rating scales. They were also invited to name other rating scales and the extent to which they used them. Non responders were sent a reminder. Characteristics of responders, such as subspecialty, age, qualifications, years in psychiatry, type of hospital, whether tutor or MRCPsych examiner, were elicited.

\section{Findings}

Ninety-nine consultants were surveyed and 73 questionnaires $(\mathbf{7 4} \%)$ were returned. The most frequently used tests were:- Hamilton Depression Rating Scale (HAM-clinician rated depression scale), Beck Depression Inventory (BECK - self rated depression scale), Wechsler Adult Intelligence Scale (WAIS general intelligence test administered by psychologist), Ravens Coloured Progressive Matrices (RAV-general intelligence test administered by psychologist), Millhill Vocabulary Scale (MIL general intelligence test psychologist administered), Minnesota Multiphasic Personality Inventory (MMPI - self-administered questionnaire rating personality indices), Clifton Assessment Procedure for the Elderly (CAPE - nurse rated functional assessment), General Health Questionnaire (GHQ - self-administered psychological screening questionnaire), Eysenck Personality Questionnaire (EPQ - self administered questionnaire rating personality indices), Hospital Anxiety and Depression Scale (HAD - self-administered anxiety and depression scale) (See Fig. 1).

Of the tests in the questionnaire, the least frequently used were the: Sandoz Clinical Assessment Geriatric (clinician rated mental state and functional assessment) used by 1 consultant, Montgomery Rating Scale (clinician rated schizophrenia scale) 1, Bulimic Investigatory Test, Edinburgh (BITE - self rated builimia scale) 2, Carroll Rating Scale for Depression (self rated depression scale) 2, Inglis Paired Associate Learning Test (psychologist administered quantitative estimate of memory 
330

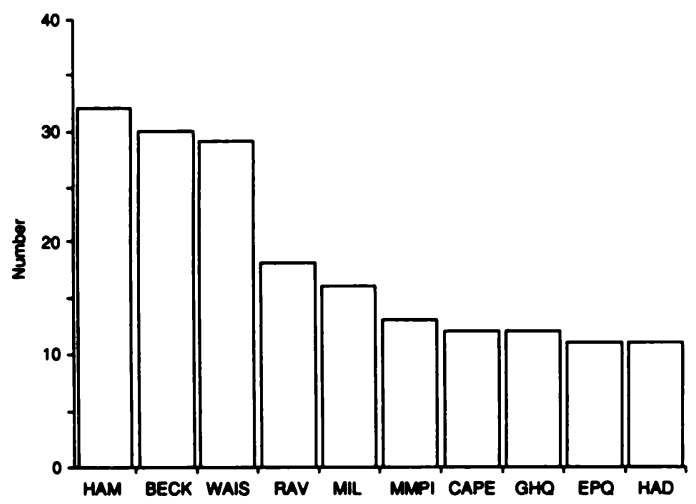

Fig. 1. Number of consultants using scales either sometimes or regularly.

impairment) 3, Michigan Alcohol Screening Test (MAST-clinician rated screening test for alcohol abuse) 4, Life Satisfaction Scale (self-administered scale appraising quality of life) 4 , Walton Black New Word Learning Test (psychologist administered quantitative estimate of memory impairment) 4.

Characteristics of users of the five most popular scales were explored using the following variables: subspeciality, general adult v. other; qualifications, M/FRCPsych v. others; age, $<39$ v. $>40$; years in psychiatry, <19v. $>20$; type of hospital, university v. others; examiner status, current/previous examiner v. other; tutor status, tutor v. non-tutor.

Four of these were found to be significant: Millhill - qualifications $(P<0.02)$, HAM - examiner status $(P<0.001)$, BECK - type of hospital $(P<0.05)$ and Millhill - examiner status $(P<0.05)$.

\section{Comment}

A criticism of the study is that it was carried out in only one NHS region introducing a bias towards
Rice and Donnelly

certain scales because of consultants having similar opinions or being influenced by the same academic centre.

Another criticism is the selection of the 32 rating scales from current literature for the questionnaire. To find any that might have been over-looked we asked consultants to inform us of other tests they used. Nearly 50 were cited but none popular enough to be mentioned by more than two consultants.

In the analysis of the characteristics of those using the more common scales, an attempt was made to separate out consultants from more academic settings, e.g., university hospital, tutors, examiners and those with additional qualifications. General adult psychiatrists were compared with the subspecialities, those over 39 years of age and under 20 years in psychiatry. Few of these factors were significant. Those variables that were associated may have been chance findings within the 35 analyses carried out. Our hypothesis that consultants in teaching centres are more likely to use rating scales was disproven. There was no marked difference between teaching and other centres. Nevertheless candidates being examined by South West Thames examiners would be advised to acquaint themselves with the HAM and Millhill.

The study shows that three scales are used much more commonly than others, the HAM, BECK and WAIS. This suggests that most consultants keep to a few well-known scales even though such a variety exists.

\section{References}

KUPFER, D. J. \& RUSH, A. J. (1983) Recommendations for depression publications. British Journal of Psychiatry, 143, 638-639.

A full list of the rating scales used and references is available from the authors on request. 